\title{
Effect of the surface model on the theoretical description of the chemisorption of atomic hydrogen on $\mathrm{Cu}(001)$
}

D. Domínguez-Ariza ${ }^{1}$, C. Sousa ${ }^{1}$, N. M. Harrison ${ }^{1,2}$, M. V. Ganduglia-Pirovano ${ }^{3}$ and F. Illas $^{1}$

1) Departament de Química Física i Centre especial de Recerca en Química Teòrica, Universitat de Barcelona i Parc Científic de Barcelona, C/ Martí i Franquès 1, E-08028 Barcelona, Spain

2) Department of Chemistry, Imperial College of Science, Technology and Medicine, London, SW7 2AY, UK

3) Fritz-Haber-Institut der Max-Planck-Gesellschaft, Faradayweg 4-6, D-14195, Berlin, Germany \& Humboldt-Universität zu Berlin, Institut für Chemie, Unter den Linden 6, D-10099 Berlin, Germany

Version August, 5, 2002

\begin{abstract}
.-
Adsorption at surfaces can be modelled using a periodic supercell approach or using finite clusters. For many systems and properties these models are complementary and often the most productive way to work is to use a combination of these techniques. If reliable data is to be obtained it is essential that convergence is achieved with respect to the size of supercell and cluster. This work discusses the convergence of chemisorption properties of $\mathrm{H}$ on $\mathrm{Cu}(001)$ with respect to the cluster size. To this end calculations of the $\mathrm{H}$ binding energy and equilibrium distance, are reported for cluster models of increasing size containing up to 77 metal atoms. Likewise, periodic slab model calculations are used to provide the corresponding values towards which the cluster approach should converge. In many previous studies of a wide variety of systems it has been established that computed equilibrium distances converge rapidly with respect to cluster size. Here, a systematic study of the dependence on cluster size shows that, for adsorption in the 4-fold site, convergence is not achieved even for very large clusters. The reason for this poor convergence is seen to be the inability of the cluster model to reproduce accurately the charge density and electrostatic potential of the crystalline surface.
\end{abstract}




\section{INTRODUCTION.-}

The chemisorption of atomic and molecular hydrogen on metal surfaces provides one of the simplest systems of interest in surface science and in heterogeneous catalysis. Several studies exist that involve low indexes $\mathrm{Cu}$ surfaces. In particular, the adsorption energy of atomic hydrogen on $\mathrm{Cu}(001)$ has been reported to be of ca. $56 \mathrm{kcal} / \mathrm{mol}^{1}$. Likewise, High-Resolution Electronic Energy Loss Spectroscopy, HREELS, experiments ${ }^{2}$ have shown that, at low coverage, the vibrational frequency of the adsorbed $\mathrm{H}$ is $565 \mathrm{~cm}^{-1}$. This study also concludes that the adsorption takes place on the four-fold hollow site. The preference for hollowsites has also been confirmed by recent HREELS experiments carried out for atomic hydrogen on $\mathrm{Cu}(111){ }^{3}$

The apparent simplicity of this system together with its relative high symmetry has motivated a number of previous theoretical studies at various levels of approximation using clusters of varying size designed to model the $\mathrm{Cu}(001)$ surface. Madhavan and Whitten ${ }^{4,5}$ used a configuration interaction, CI, method and three different $\mathrm{Cu}(001)$ cluster models, namely $\mathrm{Cu}_{9}(4,5), \mathrm{Cu}_{25}(12,9,4)$ and $\mathrm{Cu}_{33}(15,12,6)$, where the figures in parenthesis indicate the number of atoms per layer. A localization based embedding technique was further used to minimize the effects due to the limited representation of the metallic surface. Even using this sophisticated approach, the results obtained appear not to be well converged with respect to the cluster size. For the $\mathrm{Cu}_{9}$ cluster they found $\mathrm{H}$ to be located in the surface plane and an adsorption energy of $77 \mathrm{kcal} / \mathrm{mol}$. For $\mathrm{Cu}_{25}$ the equilibrium distance of $\mathrm{H}$ is predicted to be 0.84 $\AA$ above the surface plane and the adsorption energy decreases to $54 \mathrm{kcal} / \mathrm{mol}$. Finally, for the $\mathrm{Cu}_{33}$ cluster the equilibrium distance to the surface increases to $0.97 \AA$ whereas the binding energy becomes $53 \mathrm{kcal} / \mathrm{mol}$. Flad et al. ${ }^{6}$ used very small clusters, $\mathrm{Cu}_{4}$ and $\mathrm{Cu}_{5}(4,1)$, to model the $\mathrm{Cu}(001)$ surface. These authors performed unrestricted Hartree-Fock (HF) calculations and included electron correlation effects within density-functional theory (DFT). For the $\mathrm{Cu}_{4}$ cluster model they found that $\mathrm{H}$ is in the surface plane and the calculated adsorption energy is $39 \mathrm{kcal} / \mathrm{mol}$, while for the $\mathrm{Cu}_{5}$ cluster model $\mathrm{H}$ is $0.96 \AA$ out of the plane and the adsorption energy is 44 $\mathrm{kcal} / \mathrm{mol}$. Mattsson et al. ${ }^{7}$ carried out a systematic study of the influence of the cluster size on the description of the interaction of chemisorbed $\mathrm{H}$ on $\mathrm{Cu}(001)$. They performed $\mathrm{HF}$ and $\mathrm{CI}$ calculations and used several clusters - $\mathrm{Cu}_{5}(4,1), \mathrm{Cu}_{9}(4,5)$ and 
$\mathrm{Cu}_{25}(12,9,4)$ - as surface models. At the $\mathrm{CI}$ level the binding energy varies between $44\left(\mathrm{Cu}_{9}\right)$ and $51\left(\mathrm{Cu}_{25}\right) \mathrm{kcal} / \mathrm{mol}$, not too far from the experimental value. However, the calculated equilibrium distance from the surface plane, $\mathrm{R}_{\perp}$, oscillates significantly with the cluster size, e.g. $1.30 \AA$ for $\mathrm{Cu}_{5}$ versus $0.96 \AA$ for $\mathrm{Cu}_{9}$. Ricart et al. ${ }^{8}$ using a multireference configuration interaction approach and the $\mathrm{Cu}_{5}(4,1)$ cluster model, reported a value of $1.37 \AA$ for $\mathrm{R}_{\perp}$ and a binding energy of $31.5 \mathrm{kcal} / \mathrm{mol}$. Similar values were also reported by Triguero et al. ${ }^{9}$ at the Modified Coupled Pair Functional (MCPF) and DFT levels of theory also using the $\mathrm{Cu}_{5}-\mathrm{H}$ cluster. They obtained $\mathrm{R}_{\perp}$ $=1.20 \AA$ and $1.24 \AA$ at the DFT and MCPF level, respectively, and corresponding adsorption energies of $43 \mathrm{kcal} / \mathrm{mol}$ (MCPF) and 48 (DFT) kcal/mol.

From the discussion above, it is clear that for $\mathrm{H}$ adsorption in the 4-fold hollow site of $\mathrm{Cu}(001)$ the results obtained are strongly dependent on the size of the cluster model used to represent the metallic $\mathrm{Cu}(001)$ surface. At first sight this is not surprising as poor convergence of binding energies has been observed for other adsorbates. ${ }^{10}$ However, the striking feature of the $\mathrm{H}$ on $\mathrm{Cu}(001)$ system is that while the oscillations in the calculated binding energy are quite small the variations in $R_{\perp}$ are large and, as is demonstrated below, do not converge even for clusters of 77 atoms. This is unexpected as the equilibrium geometries and vibrational frequencies of adsorbed species have, in a wide variety of systems, been found to be local properties that can be adequately predicted by a cluster model. ${ }^{11,12}$ The strong variation of the equilibrium geometry with cluster size casts reasonable doubts on the use of a cluster model representation of a metallic surface even to predict structural data, at least for this particular system. A widely used alternative to the cluster model approach is the use of a slab representation of the surface, which is periodic in the two dimensions of the surface plane and finite perpendicular to the surface. This approach has been used to study the dissociation of $\mathrm{H}_{2}$ on $\mathrm{Cu}(001)$ but unfortunately the final geometry of the adsorbed $\mathrm{H}$ atoms has not been reported. ${ }^{13,14}$ A comparison between the two possible surface models using the same theoretical approach is necessary to assess the reliability of a given cluster model. It is very important to establish the convergence of the cluster approach as, at present, this is the only model within which explicitly correlated wave functions can be computed and thus a reliable description of, for instance, adsorbate excited states and the adsorption of charged species can be obtained. In addition one must realize that periodic approaches are 
constrained to use either HF or DFT with an inevitable dependence of the calculated results on the particular choice of the exchange-correlation functional which, in some cases, is of the order of the uncertainty introduced by the use of a cluster model. ${ }^{15,16}$ In this paper a detailed study of the adsorption of atomic hydrogen on $\mathrm{Cu}(001)$ using both cluster and periodic models of the metallic surface is reported. It is shown that the combined use of the two representations provides a rather complete description of this important surface science system.

\section{COMPUTATIONAL DETAILS}

Clusters models containing up to 77 metal atoms (Figure 1) have been used to represent the $\mathrm{Cu}(001)$ surface. The influence of the cluster size on geometry and energetics has been studied at different levels of theory. These include the wave function based HF and Møller-Plesset second-order perturbation theory (MP2) methods and DFT within the generalized gradient (GGA-PW91) ${ }^{17,18}$, and hybrid exchange $\left(\mathrm{B} 3 \mathrm{LYP}^{19}\right)$ approximations. The cluster models contain a local and an outer region. The former includes the five central atoms around the hollow site while the remaining atoms constitute the outer region. The $\mathrm{Cu}$ atoms in the local region are described by means of the small core, relativistic effective core potentials ${ }^{20}$ (ECP). The valence electrons are expanded in atom centred Gaussian Type Orbitals (GTO) with the standard double- $\zeta$ (LANL2DZ) basis set. ${ }^{20}$ In this way only 19 electrons per $\mathrm{Cu}$ atom $-3 \mathrm{~s}^{2} 3 \mathrm{p}^{6} 3 \mathrm{~d}^{10} 4 \mathrm{~s}^{1}$ - are explicitly included in the calculations. As in previous studies, ${ }^{15,21}$ the remaining copper atoms have been described using a oneelectron ECP as used earlier by Bagus et al. ${ }^{22}$ In this case only the $4 \mathrm{~s}$ electron of each $\mathrm{Cu}$ is explicitly included which is described with a $[4 \mathrm{~s}, 2 \mathrm{p} / 2 \mathrm{~s}, 1 \mathrm{p}]$ basis set. Finally, for hydrogen Dunning's double- $\zeta$ plus polarization correlation consistent basis set (ccpVDZ) is used. ${ }^{23}$ All calculations have been carried out using the spin restricted formalism.

Two different sets of periodic calculations using DFT with local density (LDA) or GGA functionals have been carried out by means of the CRYSTAL ${ }^{24}$ and the WIEN $97^{25}$ programs. The $\mathrm{Cu}(001)$ surface is modeled using a slab approach, where either a 4-layer (CRYSTAL) or a 7-layer (001) Cu slab (WIEN97) is used. However, there is an important difference between the two periodic treatments. Both are implementations based on an all-electron approach but use different basis sets. As for 
the cluster calculations, CRYSTAL uses a GTO basis set while WIEN97 uses the fullpotential linear augmented plane wave method (FP-LAPW). In addition, the CRYSTAL calculations are periodic in two dimensions whereas WIEN97 makes use of the repeated slab geometry approach with finite slabs periodically repeated in the third dimension but separated by a vacuum region corresponding to ten interlayer spacings. In both cases, $\mathrm{H}$ atoms are adsorbed on both sides of the slab. This facilitates the computation and avoids the need to use a spin polarized approach. The chemisorption of $\mathrm{H}$ atoms on $\mathrm{Cu}(001)$ is investigated at a coverage of 0.25 monolayers, $\mathrm{H}-(2 \times 2) / \mathrm{Cu}(001)$, with $\mathrm{H}$ occupying the four-fold hollow sites.

In the CRYSTAL calculations, the basis set used by Doll and Harrison in their study of the adsorption of chlorine on copper is used ${ }^{26}$ while the hydrogen atom has been described using a $5-11 G^{*}$ basis set. ${ }^{27}$ These functions have been adapted to periodic calculations and represent a compromise between accuracy and numerical instability due to overcompleteness of the basis set. An auxiliary Gaussian basis set containing even-tempered $\mathrm{s}$ and $\mathrm{p}$ symmetry functions was used to fit the exchange correlation potential while the energy functional was integrated explicitly on an atom centered grid. The copper auxiliary basis is the same used in Ref. 26 whereas for $\mathrm{H}$ it contains $9 \mathrm{~s}, 3 \mathrm{p}$ and $1 \mathrm{~d}$ functions with exponents between 60 and 0.06 , for the $\mathrm{s}$ functions, between 0.72 and 0.08 for the $\mathrm{p}$ set, and 0.10 for the dunction. These calculations have been carried out at the DFT level, using both the LDA, with DiracSlater exchange and the Perdew-Zunger correlation functional, ${ }^{28}$ and the GGA of Perdew et al. ${ }^{17}$ Following Doll and Harrison ${ }^{26}$ we use $\mathrm{k}_{\mathrm{B}} \mathrm{T}=0.272 \mathrm{eV}$ in a finite temperature DFT to facilitate the numerical integration over k-space. This is somewhat larger than the $0.1 \mathrm{eV}$ standard value used in plane-wave calculations but, as shown by Doll and Harrison, it is small enough to provide accurate results in the CRYSTAL calculations. Based also on previous work on this metal surface, ${ }^{26}$ an $(8 \times 8 \times 1)$ Monkhorst-Pack grid leading to $21 \mathbf{k}$-points in the irreducible Brillouin zone have been used. Adsorption energies computed within the Linear Combination of Atomic Orbitals (LCAO) approximation (either HF or DFT) are subject to a systematic error as the basis functions of the adsorbate help to describe the wavefunction of the surface. This, so called basis set superposition error (BSSE), is particularly acute for all-electron calculations as a small improvement in the description of core orbitals produces a relatively large contribution to the total energy. 
The magnitude of this effect is estimated here by computing the energy of the slab in the presence of the adsorbate basis functions, this is the standard counterpoise correction $^{29}$. The BSSE correction to the CRYSTAL results for adsorption in the 4fold hollow site is found to be about $8 \mathrm{kcal} / \mathrm{mol}$. This correction is applied to the adsorption energies reported below.

The WIEN97 periodic calculations employ the Perdew-Burke-Ernzerhorf, ${ }^{30}$ PBE, implementation of the generalized gradient approximation to DFT. The FPLAPW basis set is taken as follows: $\mathrm{R}_{\mathrm{MT}}{ }^{\mathrm{Cu}}=2.2 \mathrm{bohr}, \mathrm{R}_{\mathrm{MT}}{ }^{\mathrm{H}}=1.2$ bohr for the muffintin radii and the wave function inside the muffin-tins is expanded in spherical harmonics up to $l=12$. A maximum of $l=6$ is considered for the wave functions entering in the evaluation of non-spherical matrix elements. For the expansion of the density and the potential inside the spheres a maximum of $l=4$ is used. The energy cutoff for the plane wave representation in the interstitial region between the muffintin spheres is $16 \mathrm{Ry}$ for the wave functions and $256 \mathrm{Ry}$ for the potential. The Brillouin-zone integration have been performed using a $(12 \times 12 \times 1)$ Monkhorst-Pack grid for the $(1 \times 1)$ surface unit cell. These are $28 \mathbf{k}$-points in the irreducible Brillouin zone. A temperature broadening with a Fermi function is used with a broadening parameter $\mathrm{T}=0.1 \mathrm{eV}$. The core states are treated at fully relativistic level, while for valence states, scalar relativistic effects are included. It is worth pointing out that the BSSE commented above is inherent to GTO calculations but absent in the FP-LAPW method. Here, the accuracy of the calculation is defined by the plane wave cutoff. Therefore, the accuracy of the present computational setup has been assessed by repeating selected calculations increasing the plane wave cutoff in the interstitial region up to $18 \mathrm{Ry}$ and the plane wave cutoff of the potential representation from 256 to $400 \mathrm{Ry}$. The results of these tests will be discussed below.

\section{RESULTS AND DISCUSSION}

The different cluster models used to represent $\mathrm{Cu}(001)$ are constructed using the experimental lattice constant for the face centered cubic (fcc) copper crystal, $\mathrm{a}_{\mathrm{Cu}}=3.604 \AA .{ }^{31}$ However, for the periodic calculations the corresponding calculated values are used. The values obtained from CRYSTAL calculations are $\mathrm{a}_{\mathrm{Cu}}=3.53 \AA$ (LDA) and $3.63 \AA$ (GGA-PW91), ${ }^{26}$ both in fairly good agreement with experiment. Previous DFT-LDA calculations reported $\mathrm{a}_{\mathrm{Cu}}=3.55 \AA$ employing the FP-LAPW 
method. ${ }^{32}$ Here, $\mathrm{a}_{\mathrm{Cu}}$ calculated using CRYSTAL and the FP-LAPW method within the GGA-PBE and GGA-PW91 approximations are in excellent agreement. This indicates that although the two approaches use different basis sets, in both cases there is sufficiently flexibility to accurately expand the wave function and electron density.

The FP-LAPW method was used to establish the surface relaxation of the clean and $\mathrm{H}$ covered surfaces. For the clean surface a contraction of $2.96 \%$ of the outermost interlayer spacing with respect to the bulk value, $\Delta_{12} / \mathrm{d}_{0}$, and an expansion of $0.14 \%$ between the second and third layers, $\Delta_{23} / \mathrm{d}_{0}$, are obtained. The calculated surface relaxation is in agreement with other DFT-LDA calculations $(-3.10 \%){ }^{32}$ These values are close to the experimental value obtained by medium energy ion scattering (MEIS) which is of $-2.4 \%{ }^{33}$ while that deduced from Low Electron Energy Diffraction, (LEED), data is somewhat smaller $(-1.1 \pm 0.4 \%){ }^{34}$ The calculated vertical spacings of the $\mathrm{Cu}$ substrate of the $\mathrm{H}-(2 \times 2) / \mathrm{Cu}(001)$ structure indicate that the contraction of the surface is significantly reduced $\left(\Delta_{12} / \mathrm{d}_{0}=-0.93 \%\right.$ and $\left.\Delta_{23} / \mathrm{d}_{0}=-0.16 \%\right)$. This small surface relaxation justifies the use of an unrelaxed substrate for the CRYSTAL and cluster model calculations.

To facilitate the discussion of the influence of the cluster model size the calculated vertical distance from the $H$ atom and the surface plane $\left(R_{\perp}\right)$, and the adsorption energy $\left(E_{a d s}\right)$ are reported in Tables I and II. The latter is defined as

$$
\mathrm{E}_{\mathrm{ads}}=-\left\{\mathrm{E}\left(\mathrm{Cu}_{\mathrm{n}} \mathrm{H}\right)-\mathrm{E}\left(\mathrm{Cu}_{\mathrm{n}}\right)-\mathrm{E}(\mathrm{H})\right\}
$$

Within the DFT-GGA the calculated bond energies of molecules, the cohesive energy of solids, atomic and molecular adsorption energies are usually overestimated and the energy barriers for molecular reactions are underestimated. ${ }^{35}$ Typically, the DFTGGA derived atomic binding energies (see Eq. 1) are in error by about $0.25-1.0$ eV/atom. One must also notice that the combined use of a limited GTO basis and of the GGA functional may produce error cancellations. This is why the CRYSTAL result for the binding energy reported in Table III is slightly underestimated whereas the one obtained using the FP-LAPW basis is slightly overestimated. These provide the reference values to which the cluster approach should converge. $\mathrm{E}(\mathrm{H})$ is computed in the cluster and CRYSTAL calculations at the HF level using the GTO's discussed above. In the FP-LAPW calculations it is computed within the spin polarized GGAPBE approximation using the atomic program implemented in the WIEN-package. As 
$\mathrm{HF}$ is exact for $\mathrm{H}$ and the PBE is corrected for single electron atoms, in all cases an energy very close to the exact limit of $0.5 \mathrm{Ha}$ is obtained. In Table III the largest difference between the computed $\mathrm{E}_{\mathrm{ads}}$ and the experimental value is $\sim 2 \mathrm{kcal} / \mathrm{mol}$ which is within the deviations expected for the DFT-GGA approach. To assess the quality of the FP-LAPW basis set, the plane wave cutoff was increased from 16 to 18 Ry, which leads to a decrease of only $\sim 0.1 \mathrm{kcal} / \mathrm{mol}$ in $\mathrm{E}_{\mathrm{ads}}$ confirming the convergence with respect to the basis set. The use of a higher plane wave cutoff for the representation of the potential (from 256 to $400 \mathrm{Ry}$ ) resulted in similar, small, variations. The remaining small discrepancy between $E_{a d s}$ computed within CRYSTAL and the FP-LAPW may be attributed to a combination of factors including the slightly different geometries (recall that the CRYSTAL and cluster calculations are performed for the unrelaxed surface), the approximate correction for basis set superposition error described above and the use of different GGA functionals.

In the following the data established in the periodic calculations above is used to analyse the variation in $\mathrm{R}_{\perp}$ and $\mathrm{E}_{\text {ads }}$ computed within cluster models of various sizes. It is important to distinguish the qualitative picture and the quantitative description emerging from the cluster approach. All clusters predict the $\mathrm{H}$ atom to be bound to the metal surface (see Table I) with reasonable values for $R_{\perp}$. However, from a quantitative point of view there are very large variations. These variations are found irrespectively of the treatment of electronic exchange and correlation and thus arise solely from the use of the cluster model. Nevertheless, before examining the effects of the cluster model in detail, the influence of electronic correlation deserves some additional comment.

In general, the $\mathrm{HF}$ prediction for $\mathrm{R}_{\perp}$ is larger than that resulting from methods that account for electronic correlation either through second-order perturbation theory, MP2, or through the use of an exchange-correlation functional. This is mainly due to the fact that the Pauli repulsion between the electronic densities of the fragments is unscreened in the HF approximation. ${ }^{36}$ The effect of electronic correlation on the binding energy is less clear. One would expect the binding energy to increase when electronic correlation is included but for the $\mathrm{Cu}_{25}(16,9)$ and $\mathrm{Cu}_{29}(16,9,4)$ cluster models the interaction energy decreases when including the correlation contribution. This is surprising as it means that electron correlation for the clean cluster model is 
larger than for the cluster with added hydrogen. A possible explanation is the presence of near-degenerate states for these clean clusters which make the single-determinant approach inadequate to describe their electronic ground state. Thus the error inherent in the HF description of the metallic cluster introduces an artificial "correlation" contribution to the relative energies of the clean and chemisorbed systems. Within the DFT methods the computed $\mathrm{R}_{\perp}$ is insensitive to the choice of functional. For all clusters, the GGA-PW91 and B3LYP values are very similar; the GGA-PW91 values being systematically $0.03-0.05 \AA$ shorter.

Returning to the dependence of the calculated values of $R_{\perp}$ and $E_{a d s}$ on the cluster model; a significant feature is that, as found by other authors, $E_{\text {ads }}$ does not vary monotonically with the cluster size. ${ }^{7}$ In particular, and at variance with what is usually found for other systems, there is also a large dependence of $R_{\perp}$ on the cluster size. As the largest cluster studied here contains $77 \mathrm{Cu}$ atoms the computed $\mathrm{R}_{\perp}$ and $E_{a d s}$ were expected to approach those obtained with the periodic models. For the largest cluster considered the GGA-PW91 $R_{\perp}$ value of $0.67 \AA$, is very close to that obtained with the periodic approaches but it is clear from the dependence on cluster size that this is essentially fortuitous. $E_{\text {ads }}$ also varies with the cluster size and although the results that include correlation energy are grouped around $\sim 50 \mathrm{kcal} / \mathrm{mol}$, close enough to the values computed in the periodic formalisms, it shows a too strong oscillating character. The GGA-PW91 values are smaller than those corresponding to the slab models, this is again a result of the larger Pauli repulsion contribution to the binding energy in the cluster model and arises from the need to confine the cluster electron density in the cluster region. Nevertheless, it is important to point out the poor convergence of the self-consistent field procedure when using the GGA-PW91 functional within the cluster approach, this is probably a particular feature of the Gaussian code. The convergence problem persists even when using the quadratic convergence algorithm which is usually highly efficient but computationally very expensive.

In order to investigate the origin of the strong dependence of the calculated distance to the surface and the interaction energy with respect to the cluster size some additional calculations have been performed. The dependence of $\mathrm{R}_{\perp}$ on the cluster size was investigated with $\mathrm{H}$ occupying the atop site for the clusters $\mathrm{Cu}_{5}(1,4), \mathrm{Cu}_{9}(5,4)$, 
$\mathrm{Cu}_{13}(9,4)$ and $\mathrm{Cu}_{25}(9,16)$. These clusters are constructed in a similar way to those used to model adsorption in the for four-fold hollow site. The $\mathrm{Cu}$ atom bonded to the $\mathrm{H}$ and its four nearest neighbours in the first layer (except, obviously, for $\mathrm{Cu}_{5}$ ) are described by the Hay and Wadt ECP while the rest of atoms are described with the one electron ECP. $\mathrm{R}_{\perp}$ calculated with the GGA-PW91 for each cluster is $1.527,1.529,1.530$ and $1.548 \AA$, respectively which compare favourably to the value computed with CRYSTAL for the $\mathrm{H}-(2 \times 2) / \mathrm{Cu}(001)$ two dimensional slab $(1.535 \AA)$. Hence, for the atop site, cluster and periodic calculations are in quantitative agreement. These results strongly suggest that the variations of $R_{\perp}$ with cluster size encountered in the description of the adsorption of atomic hydrogen on the four-fold site (see Table I) have to be related to the ability of the cluster model to correctly describe the electron density felt by the $\mathrm{H}$ ad-atom at this site. In other words, the electron density at the different surface sites is not equally well reproduced by the different cluster models; all models reproduce the density for the atop site whereas the electron density at the four-fold site is strongly dependent on the cluster size and shape. This result is indicative of the existence of surface corrugation of the charge density and that, quite obviously, the $\mathrm{Cu}$ surface does not correspond closely to the simple jellium picture of a metal surface, in agreement with other findings. ${ }^{37}$ Previous analysis of the potential energy surface for $\mathrm{H}$ above several (111) metal surfaces shows that the adsorption properties are to a large extent determined by the electron density at the adsorption site. $^{38,39}$ This is fully consistent with the results discussed above.

The insensitivity of $\mathrm{R}_{\perp}$ to the cluster size for adsorption at the atop site results from the fact that the repulsive part of the potential energy curve is much steeper for this site than for $\mathrm{H}$ adsorption on the four-fold site. The $\mathrm{Cu}-\mathrm{Cu}$ distance in the 4-fold site is about $3.6 \AA$ thus the $\mathrm{H}$ can pass through this site only coming within $1.8 \AA$ of a $\mathrm{Cu}$ atom, quite different from the bond distance observed in the atop site (1.53 $\AA$ ). In the atop site, the geometry results from the competition between the attraction of the covalent bond and the Pauli repulsion between the electron densities of the hydrogen and $\mathrm{Cu}$ atoms. In the 4-fold site the $\mathrm{H}$ feels the Pauli repulsion from $\mathrm{Cu}$ atoms at a significantly greater bond distance and thus the potential energy surface is much flatter and very much more sensitive to an accurate treatment of the electron density well away from the atomic cores. Electron density difference maps could be used to illustrate this point but the use of a mixed pseudopotential approach for the cluster 
models and of an all-electron approach for the slabs, results in large differences in the total electron densities that mask the actual cluster-slab density difference. To avoid this problem the electrostatic potential generated by the nuclei and the electron density is used to compare the descriptions resulting from the cluster and periodic models. In addition, the effects of the cluster model are isolated from those due to the treatment of exchange and correlation by computing electrostatic potential maps using the HF charge density in both cluster and periodic calculations.

In Figure 2 the electrostatic potential is displayed for the clean surface along the [001] direction (this is perpendicular and out of the surface) for the line containing the four-fold site This plot shows that none of the clusters lead to an electrostatic potential for this site that approaches that of the slab model. Only $\mathrm{Cu}_{77}$ begins to approach the slab result but the strong variation from $\mathrm{Cu}_{65}$ to $\mathrm{Cu}_{77}$ implies that convergence has not been reached. Naively one would expect a more or less homogeneous convergence of the potential to the periodic limit. The present result shows that the convergence is far from homogeneous, depends strongly on cluster shape and that clusters of the order of 100 atoms are not large enough to fully converge the potential in the hollow site. It is worthy of note that the value of the electrostatic potential $0.6 \AA$ above the four-fold site fortuitously coincides with that of the slab model for the $\mathrm{Cu}_{9}, \mathrm{Cu}_{25}$ and $\mathrm{Cu}_{77}$ clusters. In Figure 3 the variation of the potential parallel to the surface at $z=0.6 \AA$ and along the 110 direction is plotted and it is clear that for these clusters the variation of the slab potential is reasonably well reproduced in the region around the position adopted by the $\mathrm{H}$ atom. In Figure 4 the difference in the electrostatic potential of the slab and $\mathrm{Cu}_{77}$ is plotted in a plane parallel to the surface at $\mathrm{z}=0.6 \AA$. It appears that even for $\mathrm{Cu}_{77}$ there is a significant discrepancy in the electrostatic potential above the 4-fold hollow site. The differences which occur at the edge of the cluster are simply an artefact due to the use of the one electron ECP's for the atoms in the outer region. It is clear from this analysis that the correspondence of $\mathrm{R}_{\perp}$ for $\mathrm{Cu}_{9}(4,5), \mathrm{Cu}_{25}(16,9)$ and $\mathrm{Cu}_{77}(36,25,16)$ to that computed in the slab model (Table I) is simply due to the fortuitous correspondence of the electrostatic potential at the $\mathrm{H}$ site to that of the slab calculations.

On the basis of the better convergence of the structural parameters of $\mathrm{H}$ adsorbed on the atop site in the previous discussion it was argued above that the electron density on the atop site is less sensitive to the cluster size. This is confirmed 
by the plots of the electrostatic potential along a line perpendicular to the surface through this site (Fig. 5) and a plane parallel the surface containing the site (Fig. 6). $\mathrm{Cu}_{25}$ yields a converged value for the electrostatic potential above the atop site in the region adopted by the $\mathrm{H}$ atom $(\sim 1.5 \AA)$. It is also clear from Fig. 4 that the difference in the cluster and slab potentials above the atop sites is much smaller than that for the hollow site. Although this insight does not allow one to design clusters a priori for the simulation of surface adsorption it does offer guidance for the design of an appropriate hierarchy of cluster models $\left(\mathrm{Cu}_{9}, \mathrm{Cu}_{25}, \mathrm{Cu}_{77}\right)$.

A point requiring further attention concerns the previously documented insensitivity to cluster size of the bond length of other adsorbates such as oxygen in the hollow site of $\mathrm{Cu}(001) .{ }^{40,41}$ The analysis carried out above is independent of the adsorbate and also gives insight into the performance of the cluster model for these systems. The larger Pauli repulsion between the $\mathrm{O}$ and surface electron density results in a bond distance which is dominated by the $\mathrm{O}-\mathrm{Cu}$ repulsion and hence relatively insensitive to the electrostatic potential and thus to the details of the cluster model. Clearly, the adsorption of atomic hydrogen on the four-fold site of a relatively unreactive metal such as $\mathrm{Cu}$ is no doubt one of the worse case scenarios for the cluster model approach. This is in agreement with the conclusions reached in a previous work on the adsorption of propyne of $\mathrm{Cu}(111){ }^{15}$

Finally, the computed frequency for the normal mode of atomic hydrogen vibrating perpendicular to the surface will be briefly discussed. This is a property of the chemisorbed system that can be directly compared to experiment. Unfortunately, both periodic calculations predict values of $\sim 700 \pm 30 \mathrm{~cm}^{-1}$, which are significantly different from the experimental value of $565 \mathrm{~cm}^{-1}$ predicted from EELS experiments. ${ }^{2}$ Anharmonic effects lower the computed value only by $\sim 50 \mathrm{~cm}^{-1}$ and hence they do not explain the rather large remaining discrepancy between theory and experiment. On the other hand, the cluster model results strongly oscillate with the different values in the $600-800 \mathrm{~cm}^{-1}$ interval. Clearly, both periodic and cluster approaches fail to predict an accurate value for this quantity. It is very likely that the $20 \%$ error exhibited by both periodic approaches arises from the extremely flat form of the corresponding potential energy surface. 


\section{CONCLUSIONS}

The present work reports on the adequacy of cluster models of varying size designed to describe chemisorption properties such as binding energy and bond distances for $\mathrm{H}$ adsorption at the $\mathrm{Cu}(001)$ surface. Periodic calculations are used to provide an accurate estimate of the limit to which cluster calculations, using the same formalism, should converge. The two periodic approaches used here use very different approaches and are nevertheless in excellent agreement with each other indicating a gratifying convergence with respect to basis set and all numerical tolerances.

The systematic study of the dependence of adsorption energy and geometry on cluster size shows that, for adsorption in the 4-fold site, convergence is not achieved even for clusters of order 100 atoms. The reason for this poor convergence is seen to be the inability of the cluster model to reproduce accurately the charge density and electrostatic potential of the crystalline surface. Chemisorption of the $\mathrm{H}$ atom on an almost perfect conducting metallic surface provides the worse possible scenario for the cluster model approach. It is worth pointing out that the problem of atomic $\mathrm{H}$ on a metal surface is not related to the level of theory, MP2, DFT or Hartree-Fock, but intrinsic to the lack of an electronic core in atomic $\mathrm{H}$.

A positive message resulting from the current work is that a detailed comparison between cluster and periodic calculations allows one to identify a cluster model - $\mathrm{Cu}_{25}(16,9)$ - which, although far from convergence, may be appropriate for cluster calculations due to a fortuitous reproduction of the density and potential in the region occupied by the $\mathrm{H}$ atom. This allows explicitly correlated wave function methods to be used to study this system and thus the interpretation of, for instance, the electronic spectra of adsorbed species can be attempted.

Finally it is worthy of note that the results presented here are specific to the cluster embedding scheme adopted. The cluster size effects may be significantly less pronounced if more sophisticated embedding techniques are used. For such a purpose, the procedure recently proposed by Carter et al. ${ }^{42}$ is especially well suited. This approach does also make use of a local region but this is embedded in a potential that when the local region is treated at the DFT level reproduces the slab result. Clearly, 
the proper choice of a local region is important because in this way the contribution of the embedding potential constitute a relatively small correction.

\section{ACKNOWLEDGEMENTS}

We gratefully acknowledge stimulating discussions with P. Blaha. Financial support has been provided by Spanish CICyT project BQU2002-04029-CO2-01 and, in part, by "Generalitat de Catalunya" grant 2001SGR-00043. N.M.H. acknowledges financial support of the European Community for staying in Barcelona through the Improving Human Potential contract HPRI-CT-1999-00071 held by the Centre de Supercomputació de Catalunya, CESCA, and Centre Europeu de Paral.lelisme de Barcelona, CEPBA. CRYSTAL calculations have been carried on the SP3 parallel machine of the CEPBA-IBM-Research-Institute, CIRI, of Barcelona. Part of the computer time was provided by the CESCA and CEPBA, through grants from the Fundació Catalana de la Recerca and from the Universitat de Barcelona. D. D. is indebted to the "Generalitat de Catalunya" for a predoctoral grant. 
Table I.- The computed equilibrium distance perpendicular to the surface, $\mathbf{R}_{\perp}$ (in $\AA$ ), as a function of the cluster size for a variety of different treatments of electronic exchange and correlation.

\begin{tabular}{lcccc}
\hline \multicolumn{1}{c}{ Cluster } & HF & MP2 & B3LYP & GGA-PW91 \\
$\mathrm{Cu}_{5}(4,1)$ & 1.305 & 1.147 & 1.172 & 1.128 \\
$\mathrm{Cu}_{9}(4,5)$ & 0.913 & 0.745 & 0.801 & 0.759 \\
$\mathrm{Cu}_{17}(16,1)$ & 1.166 & 1.043 & 1.060 & 1.024 \\
$\mathrm{Cu}_{21}(16,5)$ & 0.880 & 0.652 & 0.716 & 0.664 \\
$\mathrm{Cu}_{25}(16,9)$ & 0.574 & 0.429 & 0.604 & 0.626 \\
$\mathrm{Cu}_{25}(12,9,4)$ & 1.139 & 0.835 & 1.035 & 1.015 \\
$\mathrm{Cu}_{29}(16,9,4)$ & 1.099 & 0.810 & 0.991 & 0.963 \\
$\mathrm{Cu}_{49}(36,9,4)$ & 1.006 & 1.069 & 1.049 & 1.010 \\
$\mathrm{Cu}_{65}(36,25,4)$ & 1.033 & 0.950 & 0.982 & 0.937 \\
$\mathrm{Cu}_{77}(36,25,16)$ & 0.748 & 0.557 & 0.711 & 0.670 \\
\hline \hline
\end{tabular}


Table II.- The computed adsorption energy, $\mathrm{E}_{\mathrm{ads}}\left(\mathrm{kcal} \mathrm{mol}^{-1}\right)$, with respect to the separated fragments (cf. Eq. 1) as a function of the cluster size for a variety of different treatments of electronic exchange and correlation.

\begin{tabular}{lcccc}
\hline \hline \multicolumn{1}{c}{ Cluster } & HF & MP2 & B3LYP & GGA-PW91 \\
$\mathrm{Cu}_{5}(4,1)$ & 28.2 & 35.2 & 34.5 & 36.4 \\
$\mathrm{Cu}_{9}(4,5)$ & 47.3 & 58.1 & 33.3 & 44.9 \\
$\mathrm{Cu}_{17}(16,1)$ & 29.4 & 47.0 & 50.0 & 53.2 \\
$\mathrm{Cu}_{21}(16,5)$ & 49.8 & 60.6 & 66.7 & 68.5 \\
$\mathrm{Cu}_{25}(16,9)$ & 32.6 & 24.1 & 39.1 & 37.7 \\
$\mathrm{Cu}_{25}(12,9,4)$ & 16.8 & 43.3 & 42.1 & 44.2 \\
$\mathrm{Cu}_{29}(16,9,4)$ & 24.8 & 14.9 & 14.6 & 11.7 \\
$\mathrm{Cu}_{49}(36,9,4)$ & 51.9 & 62.5 & 49.4 & 52.3 \\
$\mathrm{Cu}_{65}(36,25,4)$ & 37.3 & 44.7 & 51.6 & 54.3 \\
$\mathrm{Cu}_{77}(36,25,16)$ & 31.6 & 31.6 & 47.0 & 50.1 \\
\hline \hline
\end{tabular}


Table III.- The adsorption geometry and energy obtained using different periodic approaches. The GTO results have been corrected for basis set superposition error as described in the text.

\begin{tabular}{rcc}
\hline \hline & $\mathbf{R}_{\perp} / \AA$ & $\mathbf{E}_{\text {ads }} /\left(\mathrm{kcal}^{\prime} / \mathbf{m o l}^{-1}\right)$ \\
GTO-LDA & 0.61 & 52.4 \\
GTO-GGA-PW91 & 0.60 & 53.5 \\
FP-LAPW-GGA-PBE & 0.61 & 56.7 \\
Experiment & --- & 56 \\
\hline \hline
\end{tabular}




\section{CAPTIONS FOR FIGURES}

Figure 1.- A schematic representation of the different clusters used to model the $\mathrm{Cu}(001)$ surface. The figures in parenthesis refer to the number of atoms in each layer.

Figure 2.- The electrostatic potential in the direction perpendicular to the surface and above the four-fold follow site as predicted by a series of cluster models. Values from a slab model as calculated using CRYSTAL are given for comparison.

Figure 3.- The electrostatic potential in the plane parallel to the surface, $0.6 \AA$ above the surface and along the [110] direction passing through the four-fold hollow site. Values from a slab model are given for comparison.

Figure 4.- The difference between the electrostatic potential computed in the periodic slab and the $\mathrm{Cu}_{77}$ cluster in a (001) plane (parallel to the surface) $0.6 \AA$ above the surface and centred on the four-fold hollow site. The spacing between the contours is 0.05 atomic units.

Figure 5.- The electrostatic potential in the direction perpendicular to the surface and above the atop site as predicted by a series of cluster models. Values from a slab model are given for comparison.

Figure 6.- Electrostatic potential in the plane parallel to the surface, $1.5 \AA$ above the surface and along the [110] direction passing through atop site. Values from a slab model are given for comparison. 


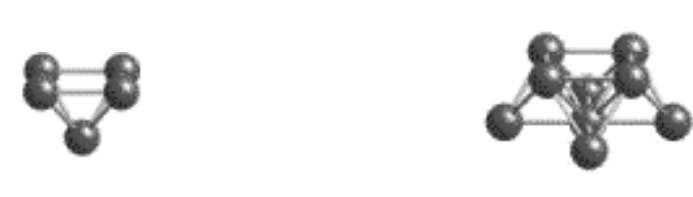

$\mathrm{Cu}_{5}(4,1)$

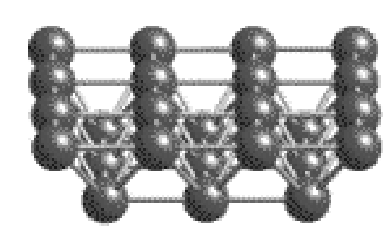

$\mathrm{Cu}_{25}(16,9)$

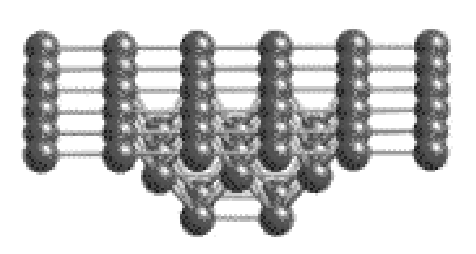

$\mathrm{Cu}_{49}(36,9,4)$
$\mathrm{Cu}_{9}(4,5)$

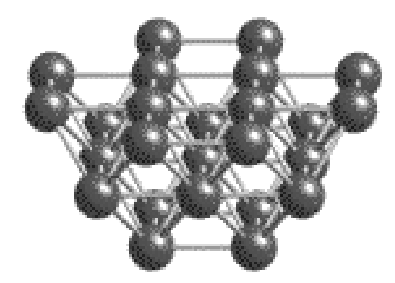

$\mathrm{Cu}_{25}(12,9,4)$

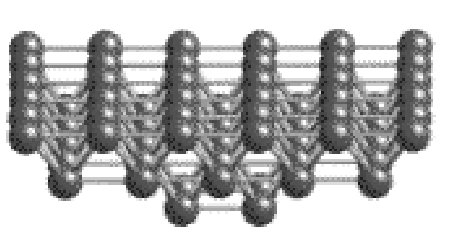

$\mathrm{Cu}_{65}(36,25,4)$

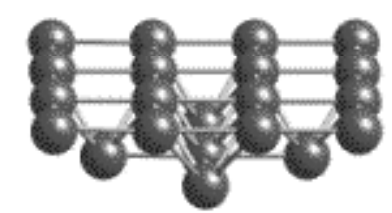

$\mathrm{Cu}_{21}(16,5)$

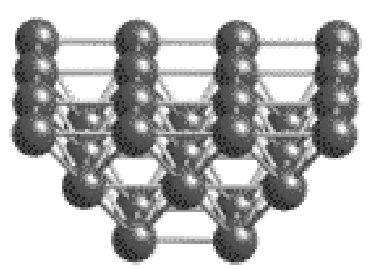

$\mathrm{Cu}_{2 g}(16,9,4)$

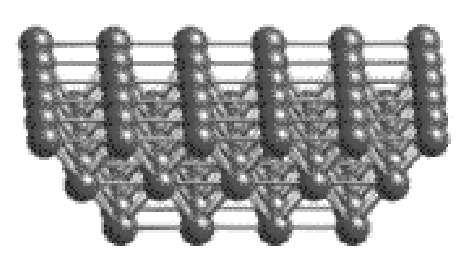

$\mathrm{Cu}_{77}(36,25,16)$

Figure 1 


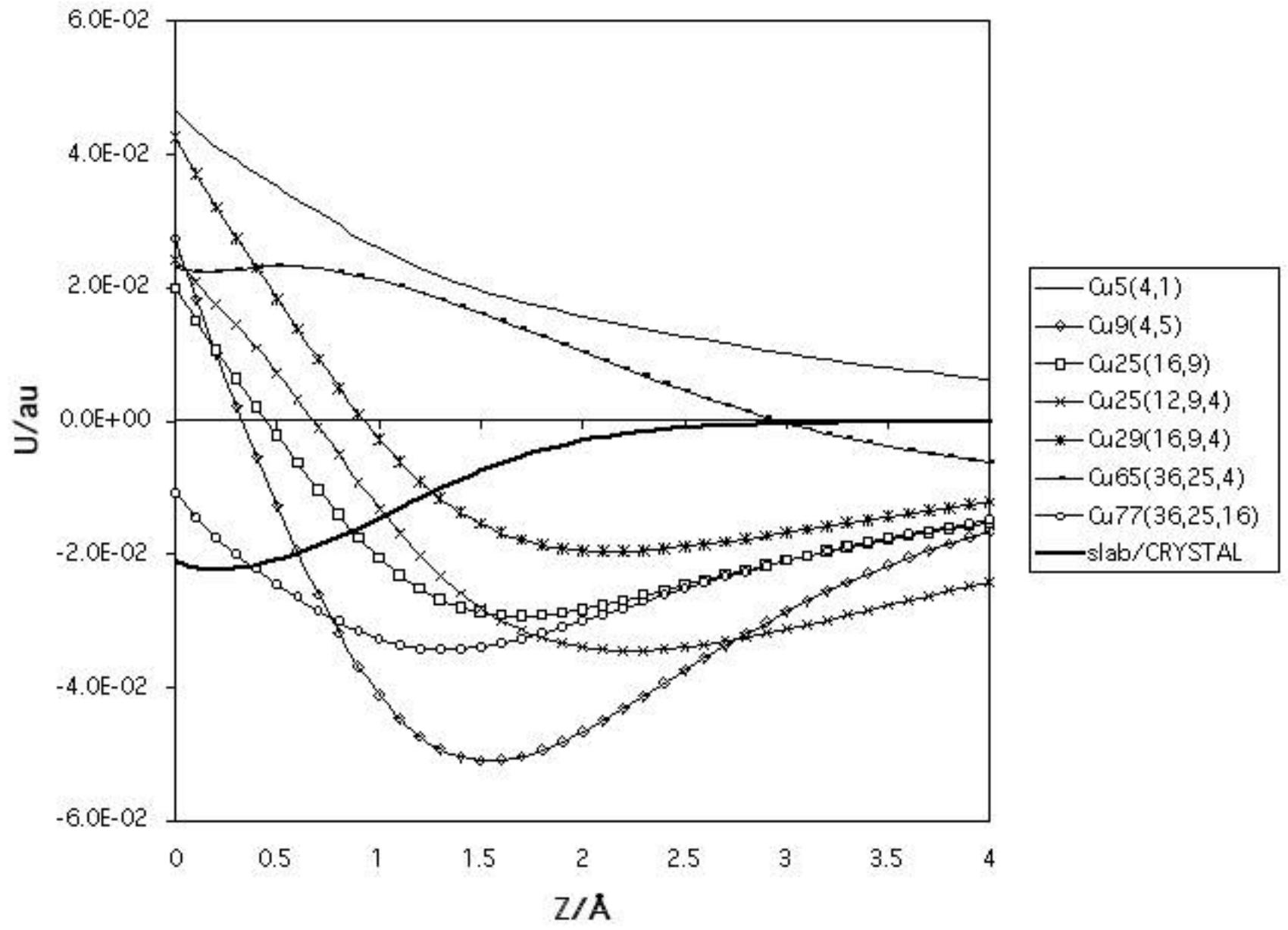

Figure 2 


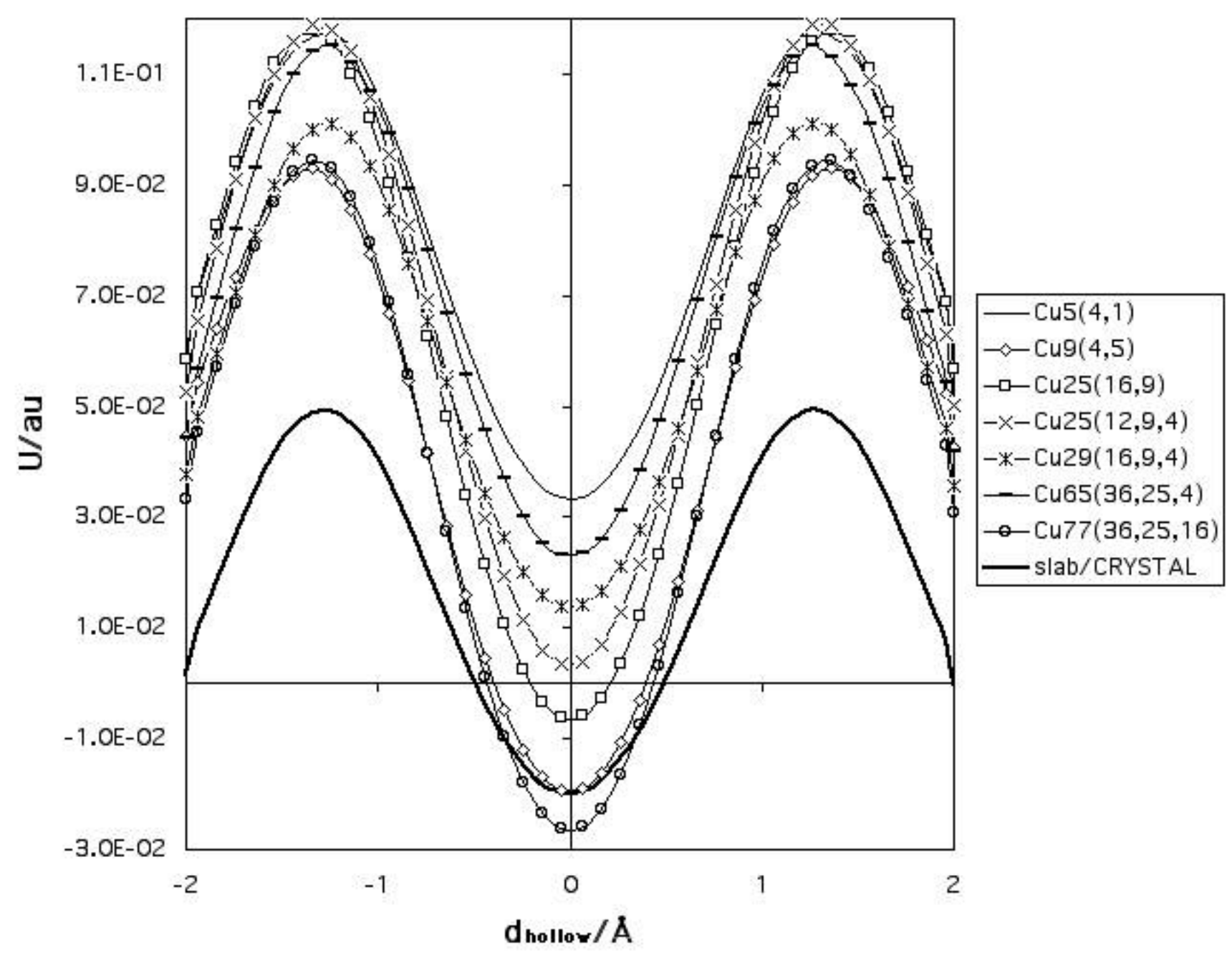

Figure 3 


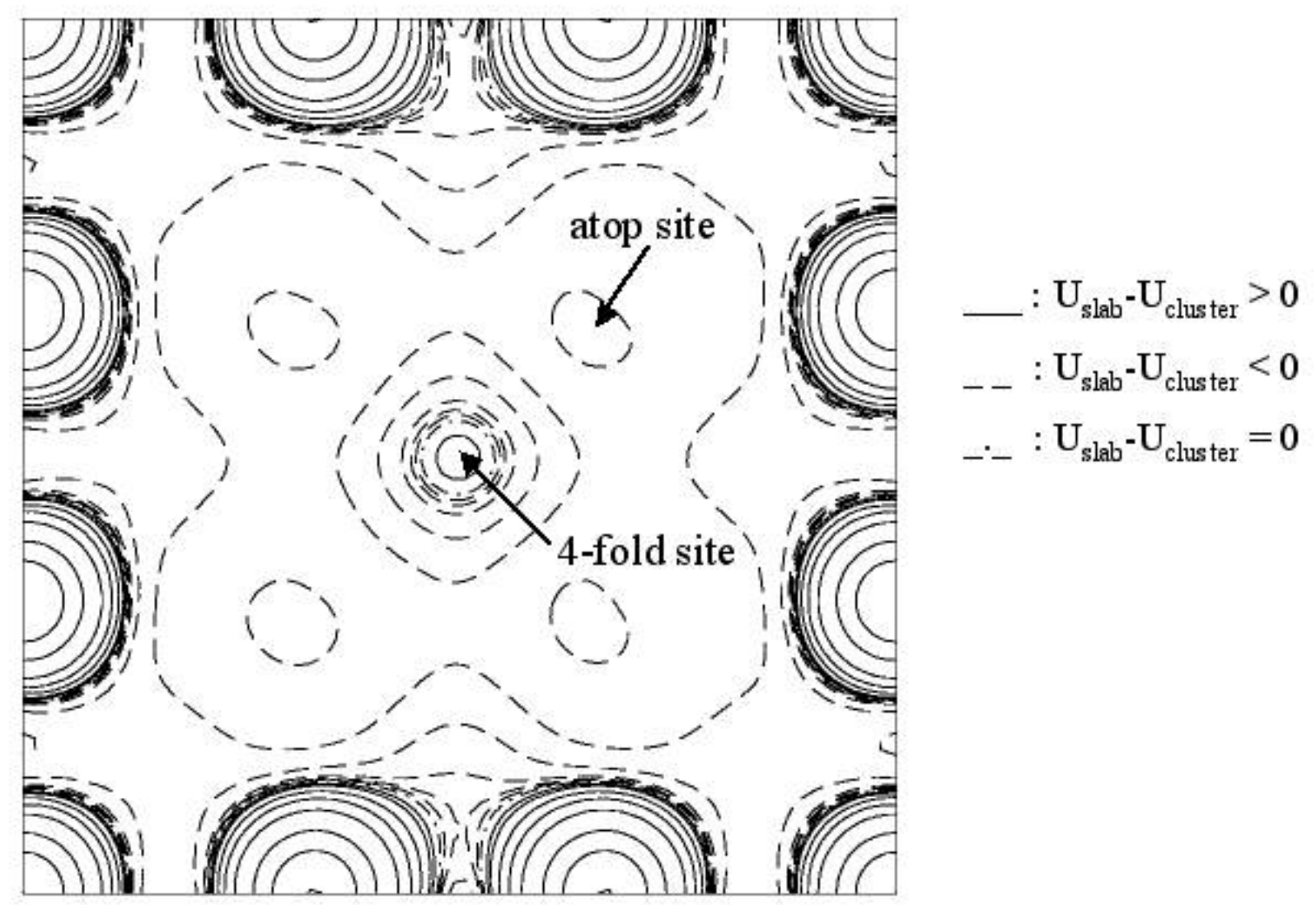

Figure 4 


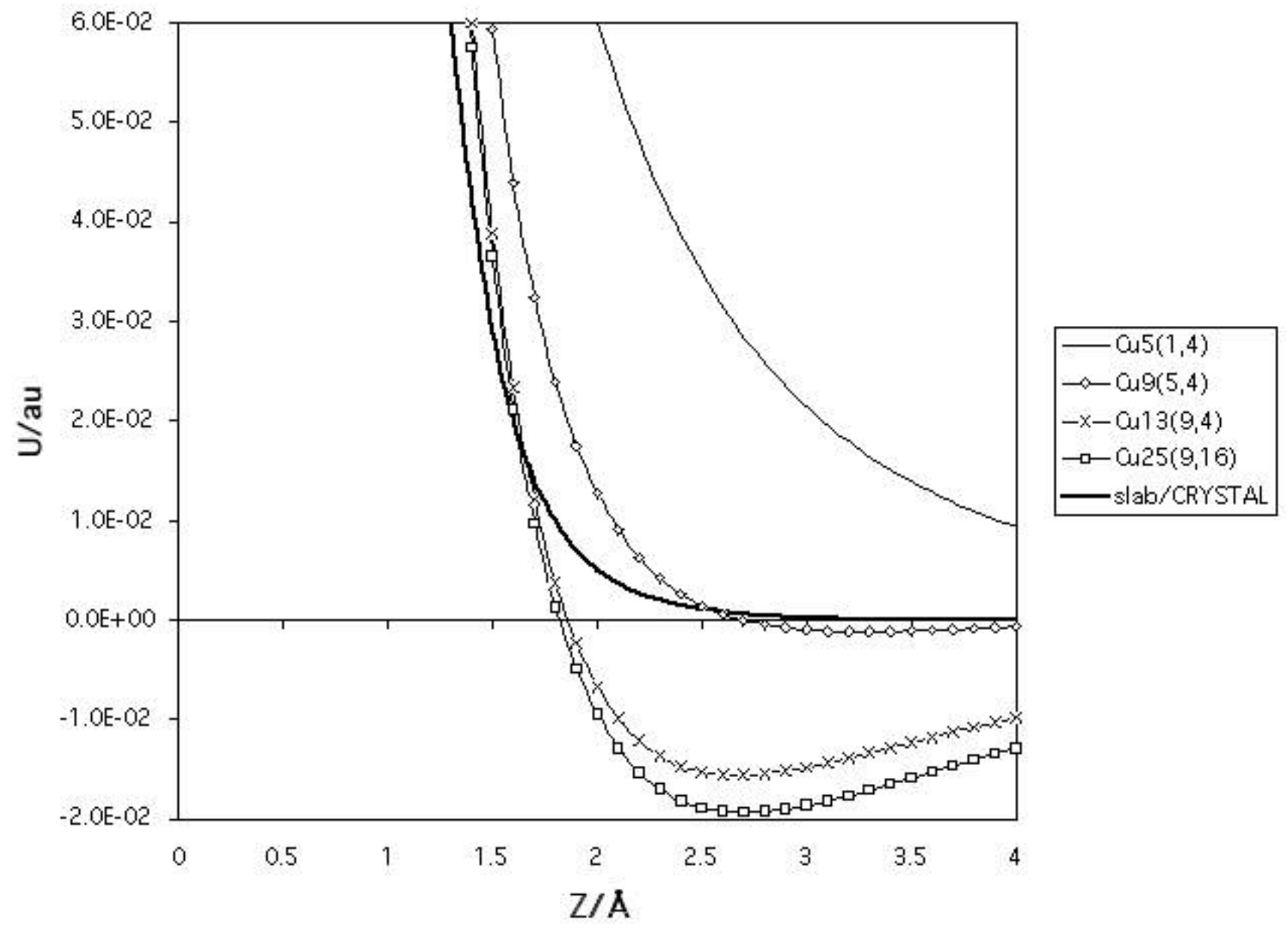

Figure 5 


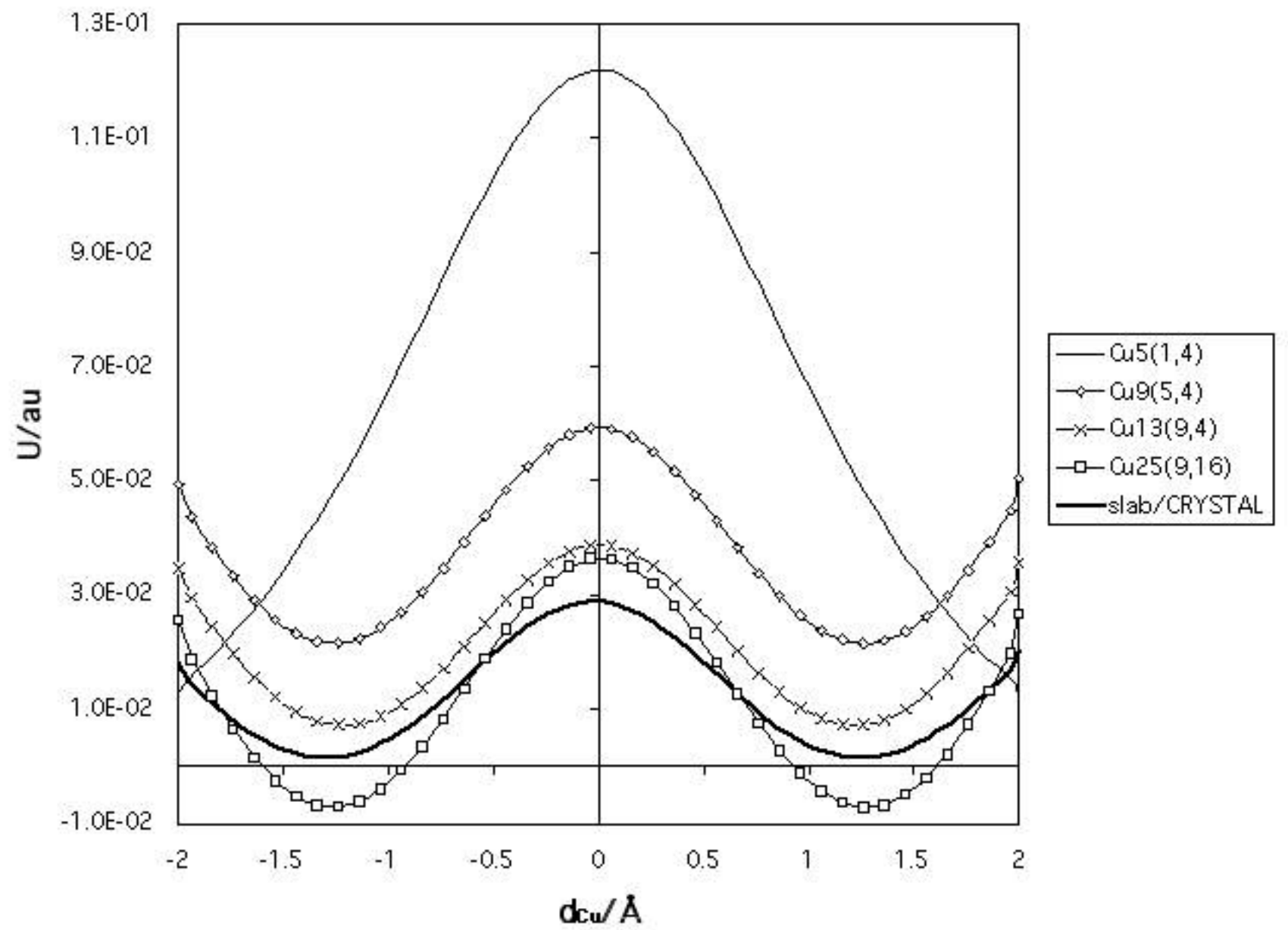

Figure 6 


\section{REFERENCES}

${ }^{1}$ G. Ertl, in the "The Nature of the Surface Chemical Bond", Edited by T.N. Rhodin and G. Ertl, North-Holland, Amsterdam 1979

${ }^{2}$ I. Chorkendorff and P.B. Rasmussen, Surf. Sci. 248 (1990) 35

${ }^{3}$ G. Lee and E. W. Plummer, Surf. Sci., 498 (2002) 229

${ }^{4}$ P. V. Madhavan and J. L. Whitten, Surf. Sci. 112 (1981) 38

${ }^{5}$ P. V. Madhavan and J. L. Whitten, J. Chem. Phys. 77(5) (1982) 2673

${ }^{6}$ J. Flad, G. Igel-Mann, M. Dolg, H. Preuss and H. Stoll, Surf. Sci. 163 (1985) 285

${ }^{7}$ A. Mattsson, I. Panas, P. Siegbahn, U. Wahlgren and H. Åkeby, Phys. Rev. B 36(14) (1987) 7389

${ }^{8}$ J. M. Ricart, A. Clotet, F. Illas and J. Rubio, J. Chem. Phys., 100 (1994) 1988-1994

${ }^{9}$ L. Triguero, U. Wahlgren, L. G. M. Pettersson, P. Siegbahn, Theor. Chim. Acta 94(5) (1996) 297

${ }^{10}$ K. Hermann, P. S. Bagus, and C. J. Nelin, Phys. Rev. B, 35, (1987) 9467

11 "The Surface Chemical Bond" by P. S. Bagus and F. Illas, in "Encyclopedia of Computational Chemistry”, P. v. R. Schleyer, N. L. Allinger, T. Clark, J. Gasteiger, P. A. Kollman, H. F. Schaefer III, P. R. Schreiner, Eds.; John Wiley \& Sons: Chichester, UK, 1998, Vol. 4, pp. 2870-2887

12 Elementary steps of catalytic processes on metallic and bimetallic surfaces", F. Illas, C. Sousa, J. R. B. Gomes, A. Clotet, J. M. Ricart, Theoretical Aspects of Heterogeneous Catalysis, Progress in Theoretical Chemistry and Physics: Vol. 8, Edited by M.A. Chaer-Nascimento, Kluwer Academic Publishers, Dordrecht, 2001, p. 149-181

${ }^{13}$ P. Kratzer, B. Hammer and J. K. Nørskov, Surf. Sci., 359 (1996) 45

14 Ž. Šljivančanin and B. Hammer, Phys. Rev. B, 65 (2002) 085414

${ }^{15}$ A. Valcarcel, J. M. Ricart, A. Clotet, A. Markovits, C. Minot and F. Illas, J. Chem. Phys., 116 (2002) 1165

${ }^{16}$ J. R. B. Gomes, F. Illas, N. Cruz Hernández, J. F. Sanz, A. Wander, N. M. Harrison, J. Chem. Phys., 116 (2002) 1684

${ }^{17}$ J. P. Perdew and Y. Wang, Phys. Rev. B, 45 (1992) 13244

18 J. P. Perdew, J. A. Chevary, S. H. Vosko, K. A. Jackson, M. R. Pederson, D. J. Singh and C. Fiolhais, Phys. Rev. B, 46 (1992) 6671

${ }^{19}$ A. D. Becke, J. Chem. Phys., 98 (1993) 5648 
${ }^{20}$ P. J. Hay and W. R. Wadt, J. Chem. Phys., 82 (1985) 299

${ }^{21}$ A. Clotet, J.M. Ricart, F. Illas, G. Pacchioni and R. M. Lambert, J. Am. Chem. Soc., 122 (2000) 7573

${ }^{22}$ P. S. Bagus, C. W. Bauschlicher, C. J. Nelin, B. C. Laskowsli and M. Seel, J. Chem. Phys., 81 (1984) 3594

${ }^{23}$ T. H. Dunning, Jr. J. Chem. Phys. 90 (1989) 1007

${ }^{24}$ V. R. Saunders, R. Dovesi, C. Roetti, M. Causà, N. M. Harrison, R. Orlando, C. M. Zicovich-Wilson, CRYSTAL 98 User's Manual, University of Torino, Torino, (1998)

25 P. Blaha, K. Schwarz and J. Luitz, WIEN97, A Full Potential Linearized Augmented Plane Wave Package for Calculating Crystal Properties, K. Schwarz, Techn. Universität Wien, Austria, 1999

${ }^{26}$ K. Doll and N. M. Harrison, Chem. Phys. Lett. 317 (2000) 282

${ }^{27}$ R. Dovesi, C. Ermondi, E. Ferrero, C. Pisani, and C. Roetti, Phys. Rev. B 29 (1984) 3591

${ }^{28}$ J. P. Perdew, A. Zunger, Phys. Rev. B 23 (1981) 5048

${ }^{29}$ S. F. Boys and F. Bernardi, Mol. Phys. 19 (1970) 553

${ }^{30}$ J. P. Perdew, K. Burke and M. Ernzerhorf, Phys. Rev. Lett., 77 (1996) 3865

${ }^{31}$ C. M. Fehrenbach, H. Bross, Phys. Rev. B, 48 (1993) 17703 and references therein

${ }^{32}$ R. Pentcheva and M. Scheffler, Phys. Rev. B, 61, (2000) 2211

${ }^{33}$ Q.T. Jiang, P. Fenter, and T. Gustafsson, Phys. Rev. B 44, (1991) 5773

${ }^{34}$ H.L. Davis and J. R. Noonan, Surf. Sci. 126, (1983) 245

${ }^{35}$ B. Hammer, L. B. Hansen, and J. Nørskov, Phys. Rev. B 59, 7413 (1999)

${ }^{36}$ A. M. Márquez, N. López, M. García-Hernández and F. Illas, Surf. Sci., 442 (1999) 463

${ }^{37}$ M. García-Hernández, P.S. Bagus and F. Illas, Surf. Sci., 409 (1998) 69

${ }^{38}$ K. Nobuhara, H. Nakanishi, H. Kasai, A. Okiji, J. Appl. Phys., 88 (2000) 6897

${ }^{39}$ K. Nobuhara, H. Nakanishi, H. Kasai, A. Okiji, Surf. Sci., 493 (2001) 271

${ }^{40}$ P.S. Bagus and F. Illas, Phys. Rev. B, 42 (1991) 10852

${ }^{41}$ J. M. Ricart, J. Torras, F. Illas and J. Rubio, Surf. Sci., 307-309 (1994) 107

${ }^{42}$ T. Kluner, N. Govin, Y. A. Wang and E. A. Carter, Phys. Rev. Lett., 86 (2001) 5954 